the group met a second time under the auspices of the Department of Urology of the Amsterdam Medical Center, Amsterdam, The Netherlands.

Methods: Medical oncologists, urological surgeons, radiation oncologists as well as pathologists from several European countries reviewed and discussed the data that had emerged since the 2002 conference, and incorporated the new data into updated and revised guidelines. As for the first meeting, the methodology of evidencebased medicine (EBM) was applied. The results of the discussion were compiled by the writing committee. All participants have agreed to this final update.

Results: The first part of the consensus paper describes the clinical presentation of the primary tumor, its treatment, the importance and treatment of testicular intraepithelial neoplasia (TIN), histological classification, staging and prognostic factors, and treatment of stage I seminoma and non-seminoma.

Conclusions: Whereas the vast majority of the recommendations made in 2004 remain valid 3 yr later, refinements in the treatment of early- and advanced-stage testicular cancer have emerged from clinical trials. Despite technical improvements, expert clinical skills will continue to be one of the major determinants for the prognosis of patients with germ cell cancer. In addition, the particular needs of testicular cancer survivors have been acknowledged.

\title{
European consensus conference on diagnosis and treatment of germ cell cancer: a report of the second meeting of the European Germ Cell Cancer Consensus Group (EGCCCG): part II
}

Krege S, Beyer J, Souchon R, Albers P, Albrecht W, Algaba F, et al.

Department of Urology, Krankenhaus Maria-Hilf, Krefeld, Germany

Eur Urol. 2008; 53: 497-513

Objectives: The first consensus report that had been presented by the European Germ Cell Cancer Consensus Group (EGCCCG) in 2004 has found widespread approval by many colleagues throughout the world. In November 2006, the group met a second time under the auspices of the Department of Urology of the Amsterdam Medical Center, The Netherlands.

Methods: Medical oncologists, urologic surgeons, radiation oncologists as well as pathologists from several European countries reviewed and discussed the data that had emerged since the 2002 conference and incorporated the new data into updated and revised guidelines. As for the first meeting the methodology of evidencebased medicine (EBM) was applied. The results of the discussion were compiled by the writing committee. All participants have agreed to this final update.

Results: The second part of the consensus paper includes the treatment of metastasised disease, residual tumour resection, salvage therapy, follow-up, and late toxicities.

Conclusions: Whereas the vast majority of the recommendations made in 2004 remain valid 3 yr later, refinements in the treatment of early-stage as well as of advanced-stage testicular cancer have emerged from clinical trials. Despite technical improvements, expert clinical skills will continue to be one of the major determinants for the prognosis of patients with germ cell cancer. In addition, the particular needs of testicular cancer survivors have been acknowledged.

\section{Editorial Comment}

A large multidisciplinary and international team of oncological specialists from Europe involved in the treatment of testicular tumors met and brought out these two consensus papers on diagnosis, staging and treatment of seminomatous and non-seminomatous testicular cancer.

The recommendations are based on evidence and on the broad clinical experience of the group and are invaluable for every urologist dealing with testicular cancer. The recommendations are clearly outlined and give 
detailed help in almost every case, from low-risk tumor with virtually $100 \%$ survival to high-risk cancer to almost $50 \%$ cure rate if treated appropriately. Again, these consensus papers are highly recommended reading.

\author{
Dr. Andreas Bohle \\ Professor of Urology \\ HELIOS Agnes Karll Hospital \\ Bad Schwartau, Germany \\ E-mail:boehle@urologie-bad-schwartau.de
}

\title{
Local progression among men with conservatively treated localized prostate cancer: results from the Transatlantic Prostate Group
}

Eastham JA, Kattan MW, Fearn P, Fisher G, Berney DM, Oliver T, et al.

Department of Urology, Memorial Sloan-Kettering Cancer Center, New York, New York, USA

Eur Urol. 2008; 53: 347-54

Objectives: Men with clinically detected localized prostate cancer treated without curative intent are at risk of complications from local tumor growth. We investigated rates of local progression and need for local therapy among such men.

Methods: Men diagnosed with prostate cancer during 1990-1996 were identified from cancer registries throughout the United Kingdom. Inclusion criteria were age $<$ or $=76 \mathrm{yr}$ at diagnosis, PSA level $<$ or $=100$ $\mathrm{ng} / \mathrm{ml}$, and, within 6 mo after diagnosis, no radiation therapy, radical prostatectomy, evidence of metastatic disease, or death. Local progression was defined as increase in clinical stage from T1/2 to T3/T4 disease, $\mathrm{T} 3$ to $\mathrm{T} 4$ disease, and/or need for transurethral resection of the prostate (TURP) to relieve symptoms $>6$ mo after cancer diagnosis.

Results: The study included 2333 men with median follow-up of 85 mo (range: 6-174). Diagnosis was by TURP in 1255 men (54\%), needle biopsy in 1039 (45\%), and unspecified in 39 (2\%). Only 29\% were treated with hormonal therapy within 6 mo of diagnosis. Local progression occurred in 335 men, including 212 undergoing TURP. Factors most predictive of local progression on multivariable analysis were PSA at diagnosis and Gleason score of the diagnostic tissue (detrimental), and early hormonal therapy (protective). We present a nomogram that predicts the likelihood of local progression within 120 mo after diagnosis.

Conclusions: Men with clinically detected localized prostate cancer managed without curative intent have an approximately $15 \%$ risk for local progression within $10 \mathrm{yr}$ of diagnosis. Among those with progression, the need for treatment is common, even among men diagnosed by TURP. When counseling men who are candidates for management without curative intent, the likelihood of symptoms from local progression must be considered.

\section{Editorial Comment}

The course of conservatively treated patients with prostate cancer is largely unknown. These patients are rarely found in clinical trials and therefore, knowledge is sparse. This multi-institutional group of authors followed a large cohort of 2333 patients for a median of 85 months and determined the rate of progression. The results are hampered by the fact that $54 \%$ of cases were detected by initial transurethral resection of the prostate, that is, by symptoms of prostate cancer. Still, the 10 -year disease-specific mortality rate was $24 \%$. Roughly, $15 \%$ of patients had progression, most within 5 years after diagnosis. Most important risk factors were high initial PSA, T3 disease and Gleason grade 4. Interestingly, early hormonal treatment was associated with significantly less progression. As the authors state correctly, these findings suggest that there is a subgroup of 